\title{
Addressing Rare-Earth Element Criticality: An Example from the Aviation Industry
}

\author{
ANTHONY Y. KU ${ }^{1,4}$ CHRISTOPHER DOSCH, ${ }^{1}$ \\ THEODORE R. GROSSMAN, ${ }^{2}$ JOSEPH L. HERZOG ${ }^{2}$ \\ ANTONIO F. MARICOCCHI, ${ }^{2}$ DREW POLLI ${ }^{3}$ and DON M. LIPKIN ${ }^{1}$

\begin{abstract}
1.-GE Global Research, 1 Research Circle, Niskayuna, NY 12309, USA. 2.—GE Aviation, 1 Neumann Way, Evandale, OH 45215, USA. 3.-Solvay, 350 George Patterson Drive, Bristol, PA 19007-3624, USA. 4.—e-mail: kua@research.ge.com
\end{abstract}

Rare-earth (RE) elements are enablers for a wide range of technologies, including high-strength permanent magnets, energy-efficient lighting, hightemperature thermal barrier coatings, and catalysts. While direct material substitution is difficult in many of these applications because of the specific electronic, optical, or electrochemical properties imparted by the individual rare-earth elements, we describe an example from the aviation industry where supply chain optimization may be an option. Ceramic matrix composite engine components require environmental barrier coatings (EBCs) to protect them from extreme temperatures and adverse reactions with water vapor in the hot gas path. EBC systems based on rare-earth silicates offer a unique combination of environmental resistance, thermal expansion matching, thermal conductivity, and thermal stability across the service temperature window. Several pure rare-earth silicates and solid solutions have been demonstrated in EBC applications. However, all rely on heavy rare-earth elements (HREEs) for phase stability. This article considers the possibility of using separation tailings containing a mixture of HREEs as a source material in lieu of using the high-purity HREE oxides. This option arises because the desired properties of RE-silicate EBCs derive from the average cation size rather than the electronic properties of the individual rare-earth cations. Because separation tailings have not incurred the costs associated with the final stages of separation, they offer an economical alternative to high-purity oxides for this emerging application.

\section{INTRODUCTION}

Rare-earth (RE) elements enable a wide range of technologies, including high-strength permanent magnets, energy-efficient lighting, high-temperature thermal barrier coatings, and catalysis. Their importance to industry has led several organizations, including the U.S. Department of Energy, the European Union, and some private companies, to classify them as "critical" materials. ${ }^{1-4}$ This designation is applied to important raw materials where concerns exist about the supply. In the case of rareearth elements, this supply risk comes from the recent concentration of the supply chain in China and was most acutely felt during a pricing bubble in $2011 .^{5}$
In recent years, there has been increasing focus on a subset of the rare-earth elements that face the tightest balance of supply and demand: europium, terbium, neodymium, praseodymium, and dysprosium. Direct material substitution for these elements is difficult because of their specific electronic, optical, or electrochemical properties. For example, the electronic band gaps of europium and terbium make them specifically suited for use in red and green phosphors, respectively. ${ }^{6}$ Similarly, dysprosium and terbium are uniquely suited to improving the high-temperature performance in $\mathrm{NdFeB}$ permanent magnets. Although these elements deserve heightened attention in the near term, it is important to recognize that technology development can radically alter the situation in the longer term. For 
instance, the introduction of material or system substitutes can mitigate some of the supply risk, while the emergence of new applications can exert upward pressure on demand.

This article considers the use of heavy rare-earth elements for an emerging application in the aviation industry, namely environmental barrier coatings (EBCs) for ceramic matrix composites (CMCs). Heavy rare-earth elements are the least abundant, making their markets especially sensitive to the introduction of new applications. However, the technical needs for the EBC application afford some flexibility in the specific rare-earth elements required and thus presents an opportunity to reduce the supply risk through creative use of the available materials. After a brief introduction of the technical requirements driving the use of heavy rare-earth elements (HREEs) in EBCs, we describe the possibility of using tailings comprising an unseparated mixture of HREEs and present experimental data showing the technical feasibility of this approach.

\section{MATERIAL SELECTION FOR ENVIRONMENTAL BARRIER COATINGS}

\section{EBC Requirements and Candidate Material Systems}

CMCs are a class of rapidly emerging materials that enable increased efficiency in turbine engines for both aviation and power generation applications. ${ }^{7}$ In aircraft engines, SiC-SiC CMCs offer the added benefit of weight savings because components made from CMCs have approximately one-third the mass of a comparable part made using a nickel superalloy. ${ }^{8}$

CMC components running in the hottest engine locations require EBCs to reduce the CMC operating temperature and to prevent recession due to reaction with water vapor in the hot gas path. ${ }^{9}$ The CMC/EBC system is shown in Fig. 1.

The key material requirements for an EBC system include: ${ }^{9}$
1. Environmental resistance under engine operating conditions. The coating must be resistant to reaction with water vapor while blocking water ingress into the underlying CMC.

2. Thermal expansion matching with the underlying CMC. Low coefficient of thermal expansion (CTE) mismatches are necessary to prevent cracking, delamination and spalling of the coating. The CTE of $\mathrm{SiC}$ and $\mathrm{SiC} / \mathrm{SiC}$ materials is between 4.5 and $5.5 \times 10^{-6} /{ }^{\circ} \mathrm{C}$.

3. Low thermal conductivity. Candidate EBC materials should have a thermal conductivity below $3 \mathrm{~W} / \mathrm{m}-\mathrm{K}$ so as to provide an effective thermal barrier between the combustion gases and the CMC.

4. Phase stability over the service temperature window. Candidate materials should be phase stable and thermochemically compatible with the underlying $\mathrm{CMC}$ up to at least $1350^{\circ} \mathrm{C}$. Crystallographic phase changes accompanied by large shear or dilational strains are especially to be avoided, as these can lead to cracking, delamination, and spallation of the coating.

EBC development has progressed through several generations of materials systems. ${ }^{10}$ Initial work through the 1990s focused on mullite, which had a good CTE match but suffered from silica volatilization at high temperatures. This issue was partially mitigated through the addition of a yttrium-stabilized zirconia (YSZ) top coat, but the multilayer coating was limited in cyclic durability because of the relatively high CTE of the zirconia. EBCs based on barium strontium aluminosilicates (BSAS) were introduced in the $2000 \mathrm{~s}$. Field tests of these coatings over thousands of hours raised concerns about the long-term stability and temperature capability of this material system. Since then, EBCs based on the rare-earth silicates have been identified as top candidates for high-temperature, high-cycle applications. ${ }^{10}$
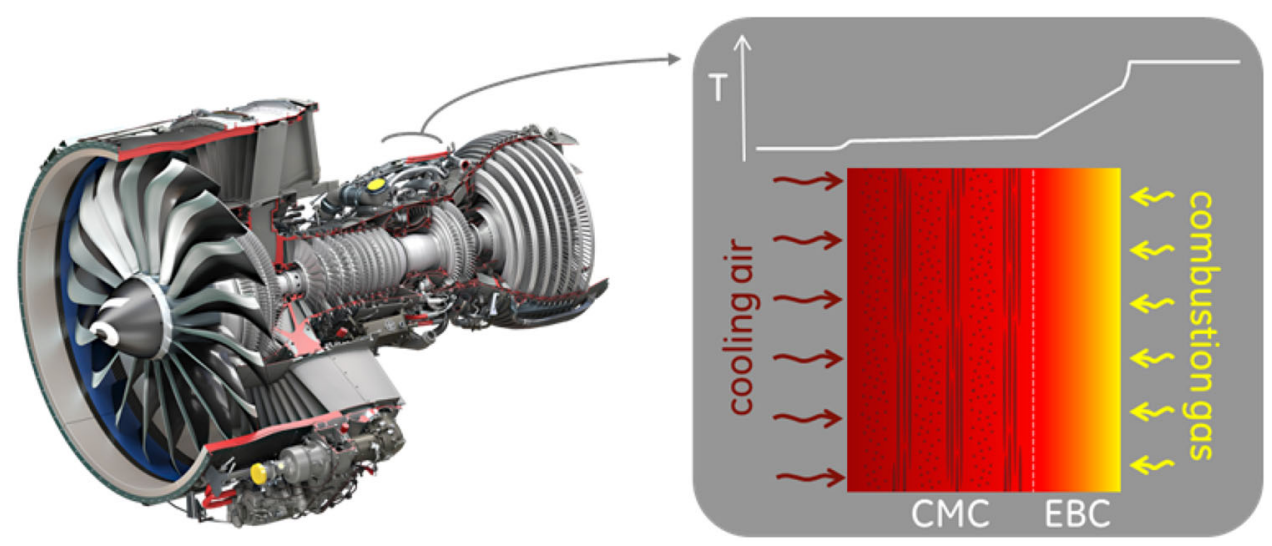

Fig. 1. Schematic of a $\mathrm{CMC/EBC}$ system in a jet engine. 


\section{Rare-Earth Silicate EBCs}

Rare-earth silicates exist as monosilicates $\left(\mathrm{RE}_{2}\right.$ $\left.\mathrm{SiO}_{5}\right)$ and disilicates $\left(\mathrm{RE}_{2} \mathrm{Si}_{2} \mathrm{O}_{7}\right)$. While the monosilicates exhibit lower silica activity and hence are more stable in high-temperature water vapor, the disilicates have a better CTE match to $\mathrm{SiC} .{ }^{11}$ Of the disilicates, $\mathrm{Y}_{2} \mathrm{Si}_{2} \mathrm{O}_{7}$ possesses perhaps the best balance of properties and affordability; however, it exhibits up to five polymorphs up to its melting point, including a Type $\mathrm{C} \mathrm{C} 2 / \mathrm{m}(\beta)$ to Type $\mathrm{D} \mathrm{P} 2{ }_{1} / \mathrm{c}$ $(\gamma)$ monoclinic phase transition between $1300^{\circ} \mathrm{C}$ and $1350^{\circ} \mathrm{C} .^{10,12}$ This phase transformation has been seen to be accompanied by exaggerated grain growth and subsequent microcracking after thermal cycling (because of the highly anisotropic thermal expansion).

In the rare-earth disilicates, the $\beta \rightarrow \gamma$ phase transition temperature seems to increase with decreasing size of the rare-earth cation, as shown in Fig. $2{ }^{13-15}$ Thus, the smallest-cation disilicates, $\mathrm{Yb}_{2} \mathrm{Si}_{2} \mathrm{O}_{7}$ and $\mathrm{Lu}_{2} \mathrm{Si}_{2} \mathrm{O}_{7}$, do not exhibit polymorphs

Table I. Chemical composition of tailings sample (balance is assumed to be oxygen)

\begin{tabular}{lc}
\hline \multicolumn{1}{c}{ Component } & At.\% \\
Rare-earth element & \\
Ho & $0.2 \%$ \\
$\mathrm{Er}$ & $0.2 \%$ \\
$\mathrm{Tm}$ & $4.2 \%$ \\
$\mathrm{Yb}$ & $74.8 \%$ \\
$\mathrm{Lu}$ & $0.1 \%$ \\
$\mathrm{Y}$ & $5.7 \%$ \\
Other rare-earth elements & $1.8 \%$ \\
Other elements & \\
$\mathrm{Ca}$ & $100 \mathrm{ppm}$ \\
$\mathrm{Fe}$ & $100 \mathrm{ppm}$ \\
\hline
\end{tabular}

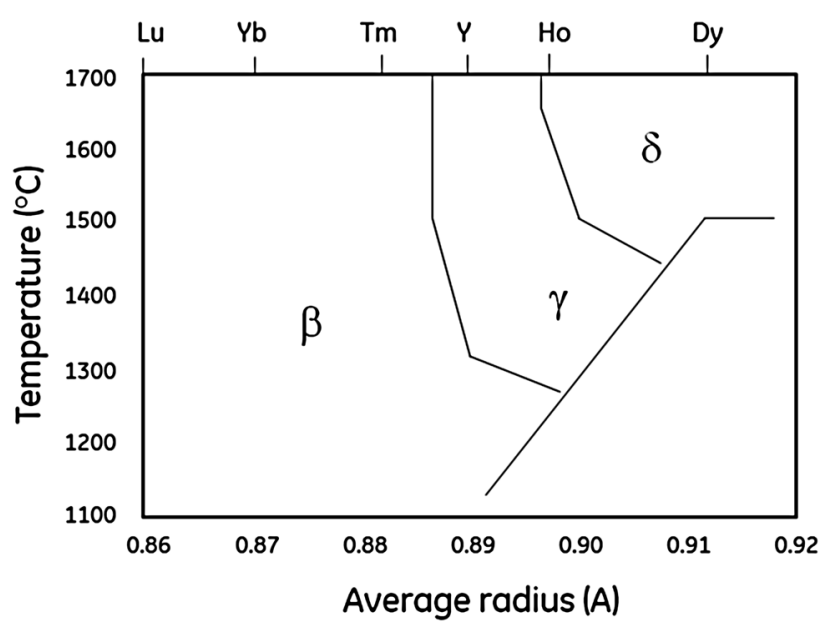

Fig. 2. Phase diagram for the rare-earth disilicates as a function of cation radius in the $+3 \mathrm{CN}_{6}$ state. ${ }^{13,14}$ Phase diagram adapted from Ref. 15. to temperatures over $1700^{\circ} \mathrm{C}$. This trend also applies to solid solutions, wherein the average ionic radius of the rare-earth elements is the key parameter governing the phase stability. ${ }^{16}$ For example, substitution of $\mathrm{Lu}$ into $\left(\mathrm{Y}_{1-x} \mathrm{Lu}_{x}\right)_{2} \mathrm{Si}_{2} \mathrm{O}_{7}$ was shown to produce solid solutions that progress from four high-temperature phase transformations between $1,225^{\circ} \mathrm{C}$ and $1,535^{\circ} \mathrm{C}$ to a single phase exhibiting stability to temperatures above $1650^{\circ} \mathrm{C} .^{15,17} \mathrm{~A}$ similar stabilizing effect was observed with increasing $\mathrm{Yb}$ substitution into $\left(\mathrm{Y}_{1-x} \mathrm{Yb}_{x}\right)_{2} \mathrm{Si}_{2} \mathrm{O}_{7}{ }^{12}$ In quantitative terms, rare-earth disilicate stability seems to require an average cation radius less than about $0.88 \mathrm{~A} .^{12}$

Based on these observations, we sought to establish whether phase stability can be demonstrated with rare-earth disilicates based on unseparated mixtures of heavy rare-earth elements from tailings, provided that the average cation radius criterion is satisfied.

\section{FEEDSTOCK SUPPLY CONSIDERATIONS}

The rare-earth separation process is shown schematically in Fig. 3. ${ }^{18}$ Rare-earth elements are mined together. Raw ores are pulverized in a beneficiation process, and the rare-earth elements are dissolved in a solvent. Individual rare earths are separated using a solvent extraction process. Lighter rare-earth elements are more abundant and typically are extracted earlier in the separation process. Heavier rare-earth elements, such as europium, gadolinium, terbium, and dysprosium follow. Yttrium is a lighter rare earth that is often separated alongside these heavier rare-earth elements because of its chemical similarity. The heaviest elements, such as $\mathrm{Tm}, \mathrm{Yb}$, and $\mathrm{Lu}$, occur at much lower concentrations and are separated at the end of the process. Given the small quantities of material and the costs needed for complete separation, these elements are commonly retained as unseparated tailings. Tailings samples that have the proper mixture of $\mathrm{Tm}, \mathrm{Yb}$, and $\mathrm{Lu}$ represent a potential feedstock for rare-earth element disilicate EBCs.

The key technical hypothesis is that tailings containing mixtures of $\mathrm{Tm}-\mathrm{Yb}-\mathrm{Lu}$ that meet the average ionic radius criterion can form disilicates without polymorphism to temperatures of at least $1500^{\circ} \mathrm{C}$.

\section{EXPERIMENTAL VALIDATION}

To test this hypothesis, a tailings sample containing a mixture of $\mathrm{Y}, \mathrm{Tm}$, and $\mathrm{Yb}$ was obtained from Solvay. The sample was chemically analyzed, mixed with an appropriate amount of silica, and pressed into pellets. These pellets were heat treated to convert them into sintered $\mathrm{RE}$ disilicate test specimens, and x-ray diffraction (XRD) was used to determine whether the samples had undergone the irreversible $\beta \rightarrow \gamma$ phase transition. 


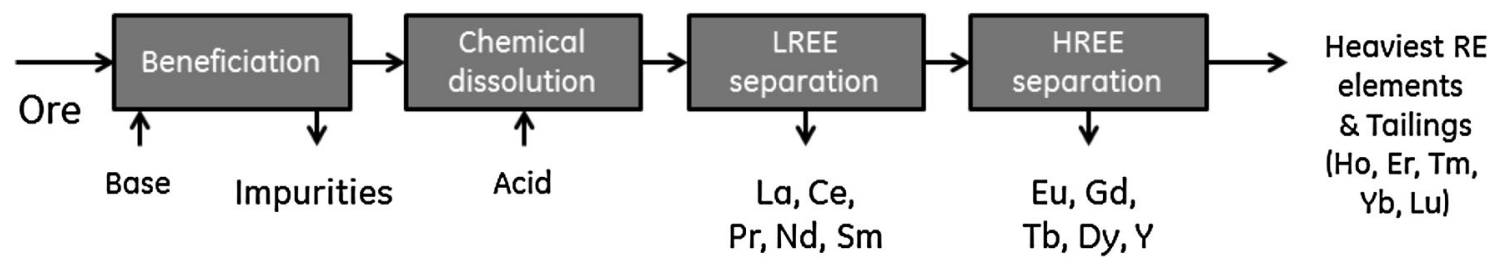

Fig. 3. Rare-earth separation process. Rare-earth elements are mined together and sequentially separated with lighter elements recovered earlier. The heaviest elements are commonly retained as unseparated tailings.

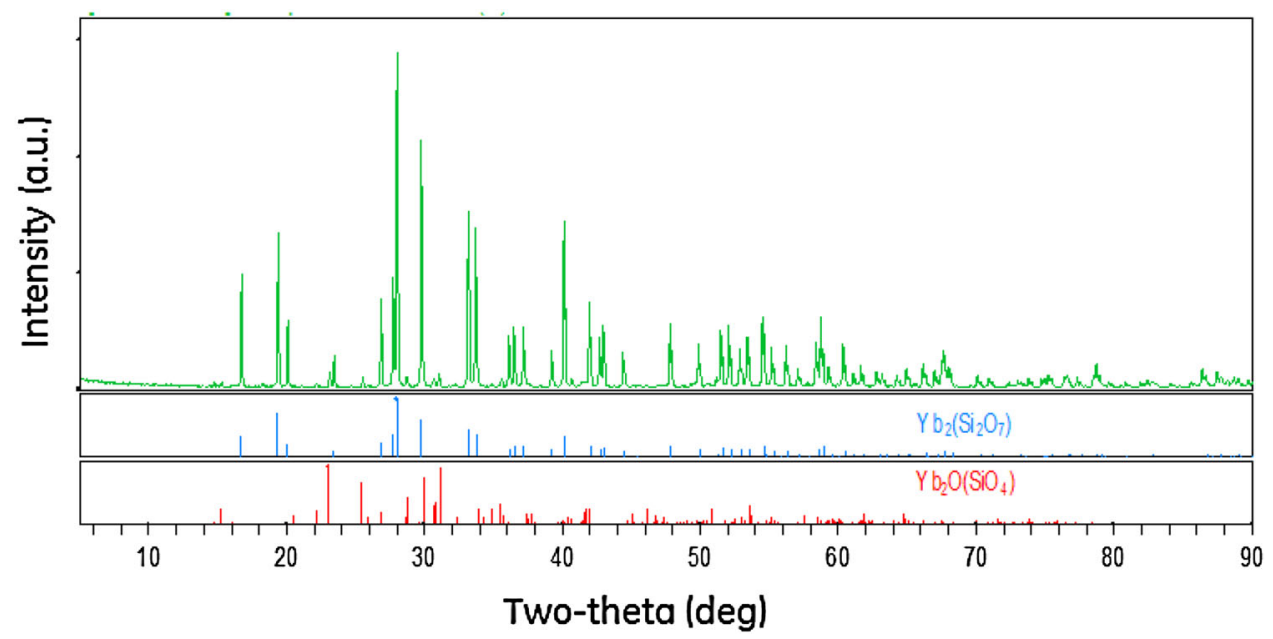

Fig. 4. XRD patterns for $\mathrm{RE}_{2} \mathrm{Si}_{2} \mathrm{O}_{7}$ prepared using tailings (green). Reference peaks for $\beta$ - $\mathrm{Yb}_{2} \mathrm{Si}_{2} \mathrm{O}_{7}$ (blue) and $\mathrm{Yb}_{2} \mathrm{SiO} \mathrm{O}_{5}$ (red) are also shown (Color figure online).

\section{Tailings Sample Composition}

The chemical composition of the tailing samples were determined using inductively coupled plasmaoptical emission (ICP-OE) spectroscopy. Table I lists the compositions of the as-received material. The tailings are enriched in $\mathrm{Yb}$ because of the enhanced natural abundance of rare-earth elements having even atomic numbers.

The average ionic size for the tailings sample can be computed from the composition and ionic radii of the constituent rare-earth elements. The cation sizes for $\mathrm{Ho}, \mathrm{Er}, \mathrm{Tm}, \mathrm{Yb}, \mathrm{Lu}$, and $\mathrm{Y}$ are $0.90 \AA, 0.89 \AA$, $0.88 \AA 0.868 \AA, 0.861 \AA$, and $0.89 \AA$, respectively ${ }_{\dot{\dot{\phi}}}{ }^{16,17}$ The weighted average ionic size is $0.87 \mathrm{~A}$, comfortably below our stability criterion of $0.88 \AA$.

\section{Pellet Processing}

The powders were mixed with silica and pressed into pellets. Fully stoichiometric $\mathrm{Yb}_{2} \mathrm{Si}_{2} \mathrm{O}_{7}$ contains 76.6 wt.\% $\mathrm{Yb}_{2} \mathrm{O}_{3}$. The target masses were: 23.1 wt. $\% \mathrm{SiO}_{2}$ and 76.9 wt.\% $\mathrm{RE}_{2} \mathrm{O}_{3}$, so as to yield a slightly rare-earth rich $\mathrm{RE}_{2} \mathrm{Si}_{2} \mathrm{O}_{7}$ (as a result, the pellets were expected to contain about $2 \mathrm{~mol} \% \mathrm{Yb}_{2}$ $\mathrm{SiO}_{5}$ ). Four pellets were pressed from homogenized powder and sintered for $4 \mathrm{~h}$ at $1550^{\circ} \mathrm{C}$ in air.

\section{Phase Stability}

The phase of the pellets was determined using XRD. The pellets were loaded with clay in a deep sample holder and scanned using a Bruker D8 Advance system (Bruker Corporation, Billerica, MA), equipped with a SOL-XE Energy Dispersive x-ray Detector. Patterns were collected over a $2 \theta$ range from $5^{\circ}$ to $90^{\circ}$ using $0.03^{\circ}$ steps with $4 \mathrm{~s}$ integration per step. The sample was rotated during data collection.

The samples made from the tailings sample powder showed majority $\beta$ phase with a minority monosilicate $\mathrm{Yb}_{2} \mathrm{SiO}_{5}$ phase detected, corresponding to less than $10 \%$ by weight (Fig. 4). No $\gamma$ phase was detected in either sample, confirming the hypothesis that phase-stable $\mathrm{RE}_{2} \mathrm{Si}_{2} \mathrm{O}_{7}$ can be produced from tailings.

\section{SUMMARY AND OUTLOOK}

A preliminary technical evaluation suggests that a phase-stable $\beta-\mathrm{RE}_{2} \mathrm{Si}_{2} \mathrm{O}_{7}$ rare-earth disilicate can be synthesized using tailings. This provides initial confirmation that mixtures of $\mathrm{Yb}, \mathrm{Tm}$, and Lu oxides can be considered as potential substitutes for the highpurity heavy rare-earth feedstock in EBC applications, thus reducing the associated supply risk. Further work is necessary to establish the effects of 
variability in the tailings and to demonstrate the ability of coatings made using tailings to meet all four of the EBC requirements.

\section{ACKNOWLEDGEMENTS}

The authors acknowledge Denise Anderson, Janell Crowder, and Eric Telfeyan for the analysis of the chemical composition of the tailings samples, James A. Brewer and Eleanor Gamble for preparation of the disilicate samples, and Steven Duclos for general discussions about rare-earth element use.

\section{REFERENCES}

1. T. Graedel, R. Barr, C. Chandler, T. Chase, J. Choi, L. Christoffersen, E. Friedlander, C. Henly, C. Jun, N.T. Nassar, D. Schechner, S. Warren, M. Yang, and C. Zhu, Environ. Sci. Technol. 46, 1063 (2012).

2. U.S. Department of Energy, 2011 Critical Materials Strategy, http://energy.gov/pi/office-policy-and-international-affairs/ downloads/2011-critical-materials-strategy. Accessed 10 Aug 2014.

3. European Commission Enterprise and Industry, Critical Raw Materials for the EU. Report of the Ad-Hoc Working Group on Defining Critical Raw Materials, 2010, http:// ec.europa.eu/enterprise/policies/raw-materials/files/docs/re port-b_en.pdf. Accessed 10 Aug 2014.
4. S.J. Duclos, J.P. Otto, and D.G. Konitzer, Mech. Eng. 132, 36 (2010).

5. U.S. Geological Survey, Rare Earth Statistics, 2014, http:// minerals.usgs.gov/minerals/pubs/historical-statistics/ds140raree.pdf. Accessed 10 Aug 2014.

6. A.M. Srivastava and T.J. Sommerer, Interface 7, 28 (1998).

7. M.C. Halbig, M.H. Jaskowiak, J.D. Kiser, and D. Zhu (Paper presented at the 51st AIAA Aerospace Sciences Meeting including the New Horizons Forum and Aerospace Exposition, Dallas, TX, 7-10 January 2013).

8. K Wood. High Perform. Comp. http://www.compositesworld. com/articles/ceramic-matrix-composites-heat-up/. Accessed November 1, 2013.

9. K.N. Lee, Surf. Coat. Technol. 133-134, 1 (2000).

10. K.N. Lee, D.S. Fox, and N.P. Bansal, J. Eur. Ceram. Soc. 25, 1705 (2005).

11. N. Maier, K.G. Nickel, and G. Rixecker, J. Eur. Ceram. Soc. 27, 2705 (2007).

12. A.J. Fernandez-Carrion, M.D. Alba, A. Escudero, and A.I. Becerro, J. Solid State Chem. 184, 1882 (2011).

13. R.D. Shannon and C.T. Prewitt, Acta Cryst. B25, 925 (1969).

14. R.D. Shannon and C.T. Prewitt, Acta Cryst. B26, 1046 (1970).

15. A.I. Becerro and A. Escudero, J. Eur. Ceram. Soc. 26, 2293 (2005).

16. N. Maier, G. Rixecker, and K.G. Nickel, J. Solid State Chem. 179,1630 (2006).

17. A.I. Becerro and A. Escudero, Chem. Mater. 17, 112 (2005).

18. N. Krishnamurthy and C.K. Gupta, Extractive Metallurgy of Rare Earths (Boca Raton, FL: CRC Press, 2004). 